\title{
DE UNA VISIÓN MONOSÉMICA A UNA VISIÓN POLISÉMICA EN LA SEMÁNTICA DE PROTOTIPOS
}

\author{
M⿳a Dolores MuÑoz NÚÑEZ \\ (Universidad de Cádiz)
}

\begin{abstract}
RESUMEN
The semantics of prototypes left to be exclusively a theory of the categorization in the standard version for to pass to analyse the different meanings of a same word and its appointment to the same or different categories. The aim of this works is to explain the reason of this change and the role which the problem of lexical polisemy plays in the revised version of the semantics of prototypes.
\end{abstract}

0 . Siguiendo la distinción entre dos etapas claramente delimitadas a lo largo de los desarrollos teóricos de la reciente semántica de prototipos que nos ofrece uno de sus principales representantes en el ámbito lingüístico europeo, G. Kleiber (1988: 1-66 y 1990), mientras que la denominada por este autor versión estándar omite, en líneas generales, cualquier consideración sobre el fenómeno polisémico y/u homonímico, ya que se presenta esencialmente como una teoría de la categorización, la versión extendida ${ }^{1}$, en cambio, sin olvidar este objetivo, supone el paso a una visión polisémica o multicategorial, que, más que explicar por qué tal entidad pertenece a una determinada categoría, intenta dar cuenta de cómo una palabra puede agrupar varios sentidos diferentes que pueden adscribirse a distintas categorías. En la base de este nuevo enfoque encontramos una

1 Se trata, según el propio autor (G. Kleiber 1990: 165), de una denominación que no aparece en la literatura sobre el tema, ya que la oposición establecida entre la versión estándar y la versión extendida no es reconocida bajo esta forma por los partidarios de una semántica del prototipo «revisada». 
concepción de la categoría diferente a la planteada en la versión estándar de esta teoría que conlleva una completa reformulación del concepto de prototipo. En lo que sigue trataremos de explicar la razón de este cambio y el papel que desempeña el problema de la polisemia léxica en la versión extendida de la semántica de prototipos.

1.1. Los planteamientos de la semántica prototípica ${ }^{2}$ en general sobre el fenómeno de la polisemia léxica son de muy distinta naturaleza a los de otras perspectivas semánticas ${ }^{3}$. En efecto, la consideración misma del significado en esta semántica surge como objeto de estudio derivado de su objetivo fundamental, que, como ya hemos indicado, no es otro que el de constituirse en una nueva teoría de la categorización.

Frente a la concepción clásica de la categorización a partir de las propiedades comunes a todos los miembros de la categoría ${ }^{4}$, la semántica de prototipos considera que ésta se constituye a partir de un ejemplo o caso óptimo, es decir, a partir de un prototipo, con respecto al cual los demás miembros se encuentran en una relación de mayor o menor grado de proximidad. De todas formas, la noción de prototipo va a cambiar a lo largo de los desarrollos teóricos de esta semántica, aunque, sobre todo, por su paso de la psicología cognitiva, donde tiene su origen, a la lingüística, y así, de ser considerado el objeto que constituye el mejor ejemplar de una categoría, o la representación mental o esquemática ${ }^{5}$ de ese prototipo-objeto, ideas calcadas de los planteamientos de E. Rosch, pasa a ser concebido como el conjunto de propiedades típicas que, esta vez, están en la base de la creación del prototipo como representación mental y del ulterior reconocimiento del prototipo-objeto, fase esta última no necesaria, sin embargo, para considerar la pertinencia del prototipo. $Y$ aún podemos tener en cuenta una más reciente concepción del prototipo, la que lo considera sólo como una 'consecuencia de la estructura de la categoría, lo que supone a la vez una nueva

2 Su origen se encuentra en los presupuestos formulados, dentro del ámbito de la psicología cognitiva, por E. Rosch (cf. 1973: 111-144, 1975: 192-233 y 1978: 27-48), E. Rosch y C. B. Mervis (1975: 573-605) y E. Rosch, C. B. Mervis, W. D. Gray, D. M. Johnson y P. Boyes-Braem (1976: 382-439). Entre sus principales representantes en el ámbito de la lingǘstica destacan W. Labov (1973: 340-373), C. J. Fillmore (1982: 31-59), G. Lakoff (1982: 1-103 y 1987), G. Kleiber (1988: 1-66 y 1990) y A. Wierzbicka (1992).

3 Cf. M. Casas Gómez y Mª D. Muñoz Núñez (1992: 134-158) y, de forma pormenorizada, Ma D. Muñoz Núñez, El problema semántico de la polisemia léxica, tesis de licenciatura defendida el 29 de junio de 1993 en la Universidad de Cádiz.

4 Véase fundamentalmente G. Kiciber (1990: 30-36) para los inconvenientes que presenta esta concepción de la categoría.

5 Según G. Kleiber (1990: 67), una de las ventajas inmediatas de la semántica prototípica es la de permitir una mayor utilización de los códigos de representaciones no verbales, hecho que, no obstante, ha sido criticado desde una óptica lingüística ya que se trataría «de una reducción inadmisible de las significaciones a imágenes $\mathrm{y}$, por tanto, de los conceptos a objetos» (E. Coseriu 1990: 244, nota 9). 
1.2. Al significado se llega ${ }^{6}$ porque las categorías -como señala E. Coseriu (1990: 242) - se denominan mediante palabras que las representan en el hablar. En tal caso, el contenido lingüístico de la unidad léxica que da nombre a una - categoría es el prototipo mismo o bien, como hemos anunciado ya, una abstracción de carácter psicológico o cognitivo que remite a él. En cuanto a los demás miembros de la categoría, presentarán un significado más o menos secundario en relación con su proximidad al prototipo. Pero así planteada tenemos que, según esta teoría, el significado de pájaro, por ejemplo, se encuentra representado, de acuerdo — según parece — con el juicio de los hablantes ${ }^{7}$, en su mejor ejemplar, gorrión, cuyo significado sería éste mismo, mientras que el de unidades como canario, jilguero, pingüino, etc. dependería de su parecido con el ejemplar típico. Se produce con ello una especie de ambigüedad en el significado de pájaro que proviene del hecho de que la «clase de denotata» tanto de este signo como de gorrión puede ser la misma, dada la casi identidad entre significado y designación en esta semántica ${ }^{8}$.

Tampoco la concepción del prototipo como conjunto de propiedades típicas difiere esencialmente, en cuanto a un análisis del significado léxico se refiere, de lo expuesto anteriormente, ya que -como señala nuevamente E. Coseriu (op. cit.: 243) - el problema que se plantea no es propiamente el del significado sino el de la inclusión de hechos u objetos en una determinada categoría, si bien es necesario identificar los prototipos con sus características básicas $y$, por tanto, los significados prototípicos con sus rasgos, que ahora, frente a lo que ocurría en la concepción anterior, pueden o no aparecer reflejados en un referente concreto. En tal caso, se observa, no obstante, cierto grado de alejamiento entre significado y referente, cuya identificación constituye, a nuestro entender, uno de los problemas centrales de la semántica prototípica. Pero el mayor grado de alejamiento se manifiesta cuando se pasa a considerar el prototipo como efecto y no como «generador» de una categoría, lo cual va a suponer una concepción del significado diferente, en estrecha conexión con la nueva concepción

6 Pero aunque la teoría de los prototipos ha tenido su más destacada aplicación en el ámbito de la semántica léxica, es necesario mencionar también los intentos de trasladar esta teoría a los ámbitos fonológico, morfológico y sintáctico fundamentalmente, en los que puede resultar satisfactorio para el análisis lingüístico adoptar una concepción no discreta de las categorías (cf., entre otros, para una panorámica de estos estudios, G. Kleiber 1990: 101-105 y J. L. Cifuentes Honrubia 1992 138-143 y 1994: 154-158).

7 Se trata de un ejemplo empleado reiteradamente en distintos trabajos dentro de esta perspectiva de análisis.

8 Recordemos que la distinción entre ambos conceptos ha sido desarrollada, fundamentalmente, en el ámbito de la semántica estructural, por E. Coseriu (cf., entre otros trabajos, 1966: 208-2: 
2.1. Esta nueva concepción de la categoría, expuesta por autores como G. Lakoff (1982) y (1987) en el ámbito de la lingüística, y a partir de la cual se hablará de versión revisada — no extendida - de la semántica prototípica, conlleva, en efecto, otra vez, una reformulación del concepto de prototipo. La razón fundamental de este cambio se encuentra precisamente - según G. Kleiber (1990: 138-146 y 149-153) - en que, con la consideración de que es el prototipomejor ejemplar el que sirve para explicar la estructuración de las categorías, no se responde satisfactoriamente al problema de la pertenencia a una categoría. Las categorías, para la versión estándar, son flexibles, de tal manera que más que hablar de pertenencia o no a una categoría hay que hablar de mayor o menor grado de pertenencia.

La concepción de la categoría defendida por la versión revisada, desprendida de cualquier exigencia de representación primera prototípica, se basa ahora en la idea de parecido de familia, es decir, en que los miembros de una categoría pueden estar ligados unos a otros sin que compartan ninguna propiedad común que defina la categoría (cf. G. Lakoff 1987: 12). Tal idea ya se encontraba en los desarrollos filosóficos de L. Wittgenstein ${ }^{9}$, en varios trabajos de E. Rosch ${ }^{10} \mathrm{y}$, consecuentemente, en los desarrollos de algunos semantistas, al menos, de orientación prototípica de la versión estándar. Pero mientras que en la versión estándar la introducción de esta idea ha tenido lugar por una asimilación con el grado de parecido con el prototipo ${ }^{11}$, en función del cual se estructura la categoría, en la versión revisada es el parecido de familia, separado de la noción de prototipo, el que organiza la estructura de la categoría. De esta manera, el proceso de pertenencia a una determinada categoría es también diferente respecto

9 Cf. Philosophical Investigations, New York, The McMillan Co., 1953, cit. por G. Kleiber (1990: 157), si bien, como señala este semantista (op. cit.: 159), el precursor de la teoría del parecido de familia es D. Stewart, Philosophical Essays, Parte II, 1818.

10 Cf., sobre todo, E. Rosch (1978: 40) y E. Rosch y C. B. Mervis (1975: 574-575), en donde señala que, al no tener la noción de prototipo un origen único, ésta ya no puede ser considerada como entidad fundadora de la estructura de la categoría, tal como se había considerado en un principio, lo que es indicativo de la nueva orientación en la concepción de la categoría.

11 Así lo ponen de manifiesto autores como S. Schlyter, «Vagheit, Polysemie und Prototypentheorie», Papers from the Institute of Linguistics University of Stockholm, 46, 1982, p. 13 , para quien hay pocas propiedades que son comunes a todos los individuos periféricos, no hay nada más que un parecido de familia o similitudes con el prototipo; D. Geeraerts, «Les données stéréotypiques, prototypiques et encyclopédiques dans le dictionnaire», Cahiers de lexicologie, 46, 1,1985, p. 29, quien considera que las distintas aplicaciones de una categoría no están ligadas entre sí por una esencia intensional común sino por parecidos de familia, y D. Dubois, La compréhension de phrases: représentations sémantiques et processus, Thèse de doctorat d'État, Paris VIII, 1986, p. 86 , autor que señala que las representaciones de los otros ejemplares se definen en relación al prototipo ya sea en términos de distancia o en términos de parecido de familia (cf. G. Kleiber 1992: 156). 
al de la semántica prototípica estándar ya que «la catégorisation se trouve justifiée par des liens d'association entre les différentes instances (ou types de référents) et non pas par un rapport entre toutes ces différentes instances et une même entité, à savoir le prototype» (G. Kleiber 1990: 159).

2.2. Bajo este punto de vista, el prototipo se presenta sólo como efecto y no como generador de la estructura de la categoría. No obstante, es necesario hablar, siguiendo a C. Fillmore (1982: 32-34) y a G. Lakoff (1987: 84-90), no de un efecto único, sino de distintos tipos de efectos a partir, bien de las relaciones entre los miembros de la categoría, bien de ciertos criterios que los distintos autores no especifican pero que no corresponden, al menos en todos los casos, como ocurría en la semántica prototípica estándar, al juicio de los hablantes. Es lo que sucede, por ejemplo, con el tipo long, señalado por C. J. Fillmore (op. cit.: 32), cuya organización categorial se presenta bajo la forma de una disyunción de condiciones en donde sólo una ocupa un lugar privilegiado, así el valor espacial de este adjetivo frente a su valor temporal. En tal caso, los mejores ejemplares son los que presentan la condición privilegiada. Algunos de estos efectos prototípicos son elegidos como tales a partir de su aplicabilidad referencial, como es el caso del tipo bachelor (cf. C. J. Fillmore, op. cit.: 34), en donde la categoría que provoca este efecto aparece definida como un conjunto de condiciones en donde los mejores ejemplares son los situados en un marco prototípico. Tal es el uso de bachelor para hacer referencia a determinado individuo, pero no para referirse, por ejemplo, al Papa, ya que este término sólo está plenamente motivado en un contex to social donde se cumplen determinadas expectativas en relación con la organización familiar ${ }^{12}$. En cuanto a los tipos señalados por G. Lakoff, basados en distintas relaciones metonímicas, estereotipos sociales, ejemplares típicos, casos ideales, parangones, generadores, submodelos y ejemplares sobresalientes, la mayoria de ellos se basan en el conocimiento del mundo en general y en el conocimiento particular de cada individuo, con lo que podemos corroborar la idea anterior de que ya no es el consenso de los sujetos hablantes sino otros criterios los que determinan unos efectos prototípicos que pueden ser sometidos a discusión en cuanto a que sean ellos los que representan a toda la categoría.

3.1. Es, pues, a partir de la reformulación de la llamada semántica de prototipos cuando tienen cabida los análisis más importantes sobre la polisemia léxica. En efecto, por una parte tenemos una nueva estructura de la categoría, basada en la idea de parecido de familia, o conexión, a veces desde el punto de

12 Una breve reflexión sobre los prototipos y su aplicabilidad referencial puede encontrarse en B. Laca (1984:9-10). 
vista del significado ${ }^{13}$, entre los distintos miembros de una categoría, y por otra el hecho - más importante aún — de que se ha asimilado la noción de prototipo a la idea de sentido básico a partir del cual se explican los otros, no a la de mejor ejemplar reconocido como tal por los hablantes. Y si ambas ideas, sentido básico y mejor ejemplar, coinciden en parte es, entre otras razones, por la propia ambigüedad de la palabra prototipo, cuya aplicación a la noción de sentido básico se ve facilitada por su acepción corriente de modelo primero a partir del cual se realizan los otros (cf. G. Kleiber 1990: 170). De esto se desprende que la polisemia léxica, para la semántica prototípica, aparece como un caso especial de categorización, en la que los diferentes sentidos de una misma palabra, ligados en mayor o menor medida, son los miembros de una categoría (cf. G. Lakoff 1987: 378), aunque cabe también la posibilidad de que se adscriban a categorías distintas. Pero así concebida, queda eliminado el problema de la distinción entre polisemia y homonimia, que sólo esporádicamente aparecían en esta teoría como dos fenómenos distintos: la homonimia como la existencia de dos o más palabras, que se pronuncian de la misma manera, con significados totalmente diferentes, $y$, por tanto, dos o más categorías, cada una de ellas con una organización prototípica distinta, y la polisemia como la existencia de una única palabra, y una sola categoría, con varios sentidos relacionados donde el principal es el prototípico mientras que los demás, considerados secundarios, corresponderán a miembros más o menos alejados de la categoría ${ }^{14}$. Por tanto, el problema se reduce ahora mayoritariamente al intento de distinción entre pertenencia de los varios sentidos de una palabra a la misma o a distintas categorias, o simplemente al análisis de la organización de esos distintos sentidos dentro de una categoría. Para ello, uno de los conceptos fundamentales manejados por algunos de los autores de la versión revisada de la semántica prototípica es el de la motivación entre esos diferentes sentidos, es decir, el de la existencia de algún vínculo entre ellos, criterio, por lo demás, tradicional en los intentos de distinción entre polisemia y homonimia. Pero, frente a la «teoría clásica», que pretendía descubrir un significado abstracto común a los varios sentidos de una palabra, se pretende ahora caracterizar la situación en que uno o más sentidos son «centrales»o «más representativos» que los demás, pudiendo estar todos ellos relacionados. Así pues, si entre los distintos sentidos existe algún vínculo estaremos ante miembros de una misma categoría; si, por el contrario, entre ellos no existe ningún tipo de vínculo, ante dos o más categorías.

13 Decimos «a veces» porque en la mayoría de los casos se habla de conexiones entre tipos de referentes o subcategorías referenciales y no entre significados, y se agrupan bajo la misma palabra que da nombre a una categoría, como vaca, por ejemplo, referentes tales como came de este animal, piel del mismo, etc. (cf. G. Kleiber 1990: 164), cuando el lexema no es en sí polisémico.

14 Cf. G. Lakoff (1982: 69 y 1987: 416). Como ejemplo de homonimia cita el de bank, «lugar donde guardamos nuestro dinero» y «porción de tierra a lo largo del borde de un río», y como ejemplo de polisemia, entre otros, el de window, «hueco en la pared» y «armazón de cristal colocado en ese hueco». 
Cuando hablamos de motivación es necesario hacer referencia a dos fenómenos fundamentales, la metáfora y la metonimia ${ }^{15}$, de los que nos interesa resaltar, siguiendo a G. Lakoff, su papel en los procesos de categorización, y, concretamente, en la formación de categorías de sentidos, que, como ya indicamos, constituyen la forma que tiene esta semántica de presentar el problema de la polisemia léxica. La metonimia adquiere un papel importante no sólo como generadora de una serie de efectos prototípicos sino también como elemento de organización de algunas estructuras categoriales, o, en la terminología del lingüista de Berkeley, modelos cognitivos idealizados (ICMs), que pueden ser de cuatro tipos (cf. G. Lakoff 1987:113-114): 1) proporcionales: especifican elementos, sus propiedades y las relaciones que los unen; 2) imágenes esquemáticas: especifican la esquematización de determinadas imágenes, como trayectorias, longitudes o formas; 3) metafóricos, en los que un dominio es comprendido en términos de otro, y 4) metonímicos, donde un submodelo es usado para comprender la categoría como un conjunto. Por tanto, por metonimia encontramos conexiones dentro de una categoria del tipo parte-todo, como se desprende del uso de los términos subrayados en las expresiones We need a couple of strong bodies (strong people) for our team y I've got a new set of wheels (cf. G. Lakoff y M. Johnson 1980: 36), productor-producto, observables en el empleo de Ford y Picasso en He bought a Ford y He's got a Picasso in his den (op. cit.: 38), lugarinstitución que allí se encuentra, tal como sucede con The White House isn't saying anything y Paris is introducing longer skirts this season (ibidem), etc.

En cuanto a la metáfora, si bien el lingüista americano no deduce de ella la serie de efectos prototípicos que había señalado para la metonimia, sí la considera, en cambio, al igual que a ésta, como uno de los elementos de organización de algunas estructuras categoriales, o ICMs. Una metáfora puede ser considerada como un trazado del ICM de un dominio al ICM de otro dominio. Este trazado define una relación entre los ICMs de ambos dominios. De esta manera, es muy común que una palabra que designa un elemento del ICM de un dominio central pase a designar también el correspondiente elemento en un ICM del dominio que se quiere comunicar. El trazado metafórico que relaciona los ICMs define la relación entre los sentidos de la palabra ${ }^{16}$, siendo considerado el sentido de la palabra del dominio central como el más básico o representativo (cf. G. Lakoff

is Cf., fundamentalmente, en la línea de esta semántica, los desarrollos teóricos que sobre estos dos fenómenos nos ofrecen G. Lakoff y M. Johnson (1980) y, exclusivamente sobre la metáfora, B. Indurkhya (1992).

16 Frente a lo que ocurre con la metonimia, o con el caso basado en correspondencias que citaremos más adelante, la metáfora pone en conexión, no elementos del mismo modelo, sino diferentes modelos cognitivos, o categorías distintas. La clave para que se hable de polisemia y no de homonimia, como en el caso señalado en la nota 14 de bank, está en que existe similitud entre los modelos en conexión, aunque en este punto G. Lakoff y M. Johnson (1980: 110-114) hablan de «Strong Homonymy», que no puede dar cuenta de las relaciones que hemos identificado en los sitemas de los conceptos metafóricos, ya que cada concepto expresado por la misma palabra es independiente, $y$ de «Weak Homonymy», en la que los varios conceptos expresados por una misma palabra pueden en muchos casos estar relacionados por similitud. 
1987: 416). Como ejemplos de este trazado podemos citar casos como los de He has a keen mind, en donde se identifica las ideas con instrumentos cortantes, $\mathbf{I}$ could feel the electricity between us, que relaciona el amor con una fuerza física, His life contained a great deal of sorrow, en donde se pone en relación la vida con un recipiente, etc. (cf. G. Lakoff y M. Johnson, op. cit.: 48-51).

$Y$ aún señala G. Lakoff (1987: 417-418) otro tipo de conexión entre sentidos, el basado en correspondencias dentro de un ICM. La palabra window puede proporcionarnos un buen ejemplo de lo que decimos. Aunque aisladamente un hueco en la pared no tiene nada en común con un armazón de cristal, por lo que no existe una razón objetiva para colocar estas dos diferentes clases de cosas en la misma categoría, el hecho de que el hueco en la pared y el armazón de cristal hayan sido producidos para adaptarse físicamente el uno al otro provee motivación para usar la misma palabra en ambos casos y en ambos sentidos, y para hacer corresponder, por tanto, estos dos sentidos a la misma categoría cognitiva, de tal manera que en expresiones como How many windows are there in your living room? la palabra windows no parece distinguir entre ambos sino que se refiere a su combinación. Hace falta expresiones como This room is too dark; we're going to have to cut a new window in that wall, This window has rotted; we're going to have to replace it, o The kids were playing ball and broke a window para encontrar los varios sentidos de este término separados. Obsérvese que incluso puede distinguirse en las dos últimas expresiones entre el armazón y el cristal, de la misma manera que sucede en español.

Cabe preguntarse ahora si con el recurso a la motivación se resuelve en lo esencial el problema de la pertenencia de las distintas acepciones de un ítem léxico a una det rrminada categoría. En este sentido es necesario resaltar que lejos de parecer novedoso, el recurso a la motivación, y, por tanto, a la similitud entre significados, ya estaba presente en semantistas anteriores, al menos desde S. Ullmann, si bien G. Lakoff parece desconocer esta circunstancia. Pero, como

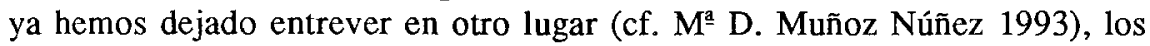
conceptos de motivación y de similitud entre los significados son demasiado relativos como para poder extraer conclusiones decisivas al respecto.

3.2. En lo que se refiere a la diferente concepción del significado propiciada por esta revisión de la semántica del prototipo, parece, en efecto, que significado y designación presentan cierta autonomía desde el momento en que, por una parte, el contenido de la unidad léxica que da nombre a una categoría ya no se identifica con el prototipo, pues éste sólo se considera ahora como un efecto o consecuencia de la estructura de la categoría, y, por otra, lo que le interesa a esta versión semántica es la consideración de que los significados relacionados de una determinada palabra (polisémica, por tanto) forman una categoría cuya organización interna se rige por el parecido entre sus miembros, parecido que 
responde a fenómenos como la metáfora, metonimia o correspondencia meramente física. De todas formas, estamos alejados de un análisis estrictamente de lengua desde el momento, sobre todo, en que el recurso único en esta teoría a las motivaciones metafóricas y metonímicas para explicar las relaciones entre significados, que, por lo demás, deberían entenderse no sólo como las conexiones existentes entre ellos sino también como su forma de funcionar en el interior de una lengua, supone el que tengamos que preguntarnos si en realidad estamos ante significados distintos $o$ ante acepciones de un mismo significado, $o$, lo que es lo mismo, ante invariantes o variantes de contenido, en donde también interviene la referencia, dando lugar a lo que podríamos llamar variantes extralingüísticas ${ }^{17}$. Además, aunque el prototipo, que sigue ocupando un papel importante en la versión revisada, se presenta como consecuencia de la estructura de la categoría, se trata en realidad de una elección bien a partir del conocimiento del mundo en general y del conocimiento particular de cada individuo, como son los casos de prototipos señalados por $\mathrm{G}$. Lakoff, bien a partir de la aplicabilidad referencial de los significados, que, en último término, remite también a un conocimiento del mundo, tal como se pone de manifiesto en los tipos de prototipos señalados por C. J. Fillmore.

3.3. En estrecha conexión con el tema de la aplicabilidad referencial y con el conocimiento general y particular del mundo se encuentra el problema de la ambigüedad que la polisemia de las palabras es susceptible de provocar en el habla, ya que el hecho de que existan situaciones o referentes más representativos que otfos podría ayudar a la desambiguación. En este sentido, hay que tener, además, en cuenta que si estamos ante invariantes distintas la organización p ototípica será también distinta; en cambio, si estamos ante variantes de una misma invariante tendremos un único prototipo. Así pues, si estamos, por ejemplo, presenciando un partido de fútbol y decimos que determinado jugador, del que conocemos su procedencia, es un Platini nos estaremos refiriendo a que es un buen jugador y no a que es descendiente de Michel Platini. O, por ejemplo, una expresión como El pájaro del prisionero canta (cit. por J. Dubois y otros 1979: 367, a propósito de las isotopías en el discurso) admite, al menos, dos interpretaciones: a) el prisionero tiene un pájaro que canta, y b) el prisionero, que es persona de determinada condición, canta. Si pensamos que uno de los sentidos de pájaro ha tenido lugar por metáfora, con lo cual existe un vínculo entre ellos, estamos ante una sola categoría con una única organización prototípica, siendo, en tal caso el significado básico el más representativo. No obstante, en este caso, como en el anterior, sólo el conocimiento extralingüístico, al margen

17 En su aplicación a la polisemia léxica, este concepto podría coincidir con el de variantes libres señalado por $\mathrm{R}$. Trujillo (1976: 182-184), y que encuentra su base en el ámbito de las connotaciones. 
de la mayor o menor representatividad de determinadas situaciones o referentes, nos permitiría saber a qué hace referencia esta expresión. En este sentido, para la semántica prototípica esta ambigüedad es superable desde el momento en que toda expresión obtiene su significado al ser asociada a un Modelo Cognitivo Idealizado determinado, que, como bien señala J. L. Cifuentes Honrubia (1994: 215), establece la relación entre la lengua y la realidad extralingüística. En efecto, a la representatividad de las situaciones y de los referentes que subyace en los tipos de prototipos señalados tanto por C. Fillmore como por G. Lakoff habría que anteponer la necesidad de un contexto y/o situación adecuados que sirva de mecanismo desambiguador, con lo que volvemos a la idea tradicional de que es el contexto en sentido amplio, unido, eso sí, a un conocimiento de la realidad, el único que puede permitir la desambiguación de expresiones, tal como ha tenido lugar en los dos casos citados anteriormente.

4. Del análisis de la semántica de prototipos en sus dos versiones se desprende, al menos, las siguientes consideraciones: la primera de ellas atañe al significado en general. Tanto en la versión estándar como en la versión revisada, uno de los problemas centrales que a nuestro entender plantea la semántica de prototipos reside en la no distinción o difusa delimitación entre significado y designación. En efecto, como hemos señalado en el análisis de la versión estándar, para esta perspectiva semántica el significado se identifica casi plenamente con la cosa, ya que, aun considerando que el prototipo se presenta como un conjunto de propiedades típicas que no tienen por qué aparecer reconocidas en ningún ejemplar concreto de la realidad, la categoría en su conjunto está constituida, no por significados, sino por referentes, que presentan ciertas características más o menos próximas a las del prototipo, pues de lo que se trata es de explicar «cómo y por qué los objetos se subsumen bajo determinados conceptos» (E. Coseriu 1990: 243). No obstante, al tratar de identificar los prototipos mismos, se identifican sus rasgos, es decir, su significado, aunque éste no se pretende describir ni analizar.

La versión revisada, en cambio, al plantear el problema de la categorización de modo diferente, se enfrenta al significado también de distinta manera, ya que es ahora el parecido de familia el que da respuesta al cómo y al por qué de la pertenencia de determinadas entidades a una u otra categoría, mientras que la noción de prototipo como elemento en torno al cual se estructura la categoría desaparece. Pero, en este sentido, es necesario hacer, a su vez, estas otras consideraciones: si bien se habla de parecido de familia entre los distintos significados o sentidos de una palabra polisémica, a través, sobre todo, de las motivaciones metafóricas y metonímicas, en muchos casos de lo que se trata es de una similitud entre referentes que, desde el punto de vista del significado, son monosémicos, tal como sucede en el caso de vaca, «animal», «piel de ese 
animal», etc. (cf. nota 13), a menos que se tengan en cuenta también en estos casos metáforas y metonimias ocasionales, que, como ya hemos indicado anteriormente, dan lugar a lo que podríamos denominar variantes extralingüísticas (recuérdese, en este sentido, la base eminentemente referencial de los hechos metonímicos y de la mayoría de los metafóricos). A esto hay que añadir el hecho, también señalado, de que los significados prototípicos que se desprenden de la nueva estructura de la categoría sólo pueden ser deducibles a partir de su aplicabilidad referencial o a partir del conocimiento del mundo general y particular de cada individuo.

En lo que se refiere a la polisemia de las palabras, los análisis de esta perspectiva semántica no parecen proporcionarnos ninguna solución al problema, no en cuanto fenómeno general del lenguaje, estrechamente conectado con el principio de economía, cuya defensa viene dada de antemano, sino como fenómeno que deba o no competir a la estructura interna de cualquier lengua. Para los semantistas prototípicos la polisemia de las palabras existe desde el momento en que el parecido de familia, responsable de la nueva estructura de la categoría, justifica su existencia. Pero nada más alejado de otros planteamientos semánticos sobre el tema, ya que - recordemos- la base de esta corriente lingüística no es el análisis del significado en cuanto tal, sino sólo como vehículo que conduce a la referencia, a partir de la cual, eso sí, se puede dar solución al problema, no de la polisemia, sino de la ambigüedad que ésta puede generar.

\section{REFERENCIAS BIBLIOGRÁFICAS}

Casas Gómez, M. y Muñoz Núñez, Mª D. (1992), «La polisemia y la homonimia en el marco de las relaciones léxicas», Wotjak, G. (ed.), Estudios de lexicología y metalexicografía del español actual, Tübingen, Max Niemeyer Verlag, pp. 134-158.

Cifuentes Honrubia, J. L. (1992), «Teoría de prototipos y funcionalidad semántica», Estudios de Lingüística, 8, 133-177.

(1994), Gramática cognitiva. Fundamentos críticos, Madrid, Eudema.

Coseriu, E. (1966), «Structure lexicale et enseignement du vocabulaire, Actes du premier Colloque International de Linguistique Appliquée, Nancy, pp. 175217.

(1990), «Semántica estructural y semántica «cognitiva»», Profesor Francisco Marsá. Jornadas de Filología, Barcelona, pp. 239-282.

Dubois, J. y otros (1979), Diccionario de lingüística, Madrid, Alianza Editorial. Fillmore, C. J. (1982), «Towards a Descriptive Framework for Spatial Deixis», R. J. Jarvella y W. Klein (eds.), Speech, Place, and Action, London, John Wiley \& Sons Ltd., pp. 31-59. 
Indurkhya, B. (1992), Metaphor and Cognition. An Interactionist Approach, Dordrecht, Kluwer Academic Publishers.

Kleiber, G. (1988), «Prototype, stereotype, un air de famille?», DRLVA, 38, pp. $1-66$.

(1990), La sémantique du prototype, Paris, P.U.F.

Labov, W. (1973), «The boundaries of words and their meanings», C. J. Bailey

y R. Shuy (eds.), New Ways of Analyzing Variation in English, Washington

D. C., Georgetown University Press, pp. 340-373.

Laca, B. (1984), «La semántica de prototipos. ¿Hacia una lingüística de las cosas?», Relaciones, 1, pp. 9-10.

Lakoff, G. (1982), Categories and Cognitive Models, Cognitive Science Program, Institute of Cognitive Studies, University of California at Berkeley.

(1987), Women, Fire and Dangerous Things. What Categories reveal about the Mind, Chicago, London, The University of Chicago Press.

Lakoff, G. y Johnson, M. (1980), Metaphors We Live by, Chicago, The University of Chicago Press.

Muñoz Núñez, Maㅡ. D. (1993), El problema semántico de la polisemia léxica, Tesis de licenciatura, Univ. de Cádiz.

Rosch, E. (1973), «On the Internal Structure of Perceptual and Semantic

Categories», T. E. Moore (ed.), Cognitive Development and the Acquisition of Language, New York, Academic Press, pp. 111-144.

(1975), «Cognitive Representations of Semantic Categories», Journal of Experimental Psychology, 104, pp. 192-233.

(1978), «Principles of Categorization», E. Rosch y B. Lloyd (eds.), Cognition an 1 Categorization, Hillsdale, Laurence Erlbaum Ass., pp. 27-48. Rosch, E. y Mervis, C. B. (1975), «Family Resemblances. Studies in the Internal Structure of Categories», Cognitive Psychology, 7, pp. 573-605.

Rosch, E., Mervis, C. B., Gray, W. D., Johnson, D. M. y Boyes-Braem, P. (1976), «Basic Objects in Natural Categories», Cognitive Psychology, 8, pp. $382-439$.

Trujillo, R. (1976), Elementos de semántica lingüística, Madrid, Cátedra.

Wierzbicka, A. (1992), Semantics, culture and cognition, New York, Oxford, Oxford University Press. 